\title{
Idiopathic Azygos Vein Aneurysm Mimicking a Mediastinal Mass: Case Report and Review of Literature
}

\author{
Khalid Mohammad $^{\mathrm{a}, \mathrm{b}}$, Nutan Bhaskar ${ }^{\mathrm{a}}$, Mohammad F Siddiqui ${ }^{\mathrm{a}}$
}

\begin{abstract}
True aneurysmal dilatation of the azygos vein is a rare entity which has been reported in literature on only a few occasions. We report a case of idiopathic azygos aneurysmal dilation that was incidentally discovered on chest roentgenogram as a mediastinal mass. A contrast enhanced CT scan of the chest showed a large azygous arch aneurysm with normal appearance of other mediastinal vasculature. She was managed conservatively with outpatient follow up. A repeat CT Chest after one year showed no change in size of the aneurysm.
\end{abstract}

Keywords: Azygos vein aneurysm; Idiopathic

\section{Introduction}

Enlargement of the azygos vein can be due to an increase in central venous pressure, portal vein hypertension, azygos continuation of the inferior vena cava (IVC), a tumor or thrombus located in the inferior vena cava, post-traumatic pseudoaneurysm and pregnancy[1, 2]. High central venous pressure is by far the most common cause and mainly results from cardiac decompensation [3]. True aneurysmal dilatation is a highly uncommon entity which has been reported on very few occasions [4-8].

\section{Case Report}

An 83-year-old Caucasian female patient was admitted to

\footnotetext{
Manuscript accepted for publication February 21, 2013

${ }^{\text {a}}$ Department of Pulmonary and Critical Care Medicine, University of Arkansas for Medical Sciences, Little Rock, AR, USA

${ }^{\mathrm{b}}$ Corresponding author: Khalid Mohammad, Department of Pulmonary and Critical Care Medicine, University of Arkansas for Medical Sciences, Little Rock, AR, USA. Email: khalidmdmdoc@gmail.com
}

doi: http://dx.doi.org/10.4021/jmc1127w the hospital with generalized weakness, one episode of hematemesis 2 weeks ago and left leg swelling, redness and pain. She had a past medical history significant for osteoarthritis and atrial fibrillation. On physical examination she was afebrile with a pulse of 106/min irregularly irregular, blood pressure of $155 / 60 \mathrm{mmHg}$, respiratory rate of $18 / \mathrm{min}$ and oxygen saturation of $98 \%$ on room air. Chest auscultation showed bilateral equal breath sounds. Cardiac auscultation was significant for an irregularly irregular S1, S2 with no murmurs. The left leg was red, swollen, warm and tender to touch. Peripheral pulses were well felt.

Initial laboratory studies demonstrated a hemoglobin and hematocrit of $4.4 \mathrm{~g} / \mathrm{dL}$ and 14.9 respectively. EKG showed atrial fibrillation. Chest roentgenogram showed a large right paratracheal mass with left lower lobe atelectasis and pleural effusion (Fig. 1). A contrast enhanced computed tomographic (CT) scan of the chest showed a large azygos arch aneurysm measuring $5.5 \times 3.4 \mathrm{~cm}$ with normal appearance of other mediastinal vasculature (Fig. 2-4). An Esophagogastro-duodenoscopy was done which showed a healing ulcer in the antrum of the stomach. A transthoracocic echocardiogram showed a normal left ventricular systolic function with mildly increased LV thickness. The right ventricular systolic function was normal. There was no laboratory or imaging data to suggest portal hypertension. A vascular surgery evaluation recommended a conservative management of the aneurysm. The patient was treated for upper gastro-intestinal

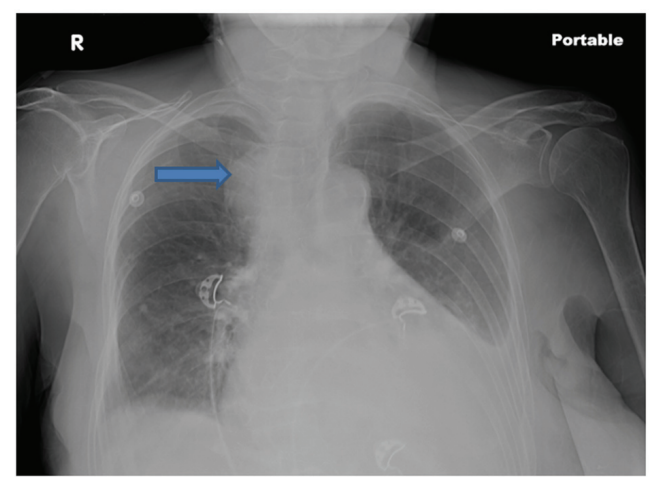

Figure 1. Chest roentgenogram showing a right paratracheal mass with left pleural effusion. 


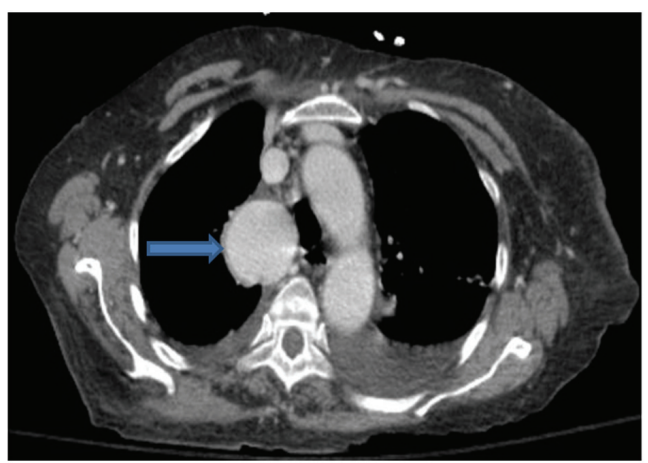

Figure 2. An axial thoracic contrast-enhanced CT image shows a well-defined $5.5 \mathrm{~cm}$ mass (thick arrow) in mediastinum. The mass enhances remarkably and homogeneously in the venous phase, with isodensity to the large vessels in the mediastinum, indicating a vascular origin.

tract bleed and lower extremity cellulitis and discharged home. She continued to remain asymptomatic and a follow up CT scan of chest showed that there was no increase in size of the azygos arch aneurysm.

\section{Discussion}

Azygos vein aneurysm was first described by Walker in 1963 as an idiopathic lesion [9]. It is often described as a fusiform, saccular, or oval-shaped mass with a diameter ranging from 2.5 to $5.0 \mathrm{~cm}$. Traumatic pseudoaneurysm is a differential diagnosis of true aneurysm [10].

Most patients with venous aneurysms are asymptomatic and the lesion is detected as an incidental finding on the chest radiograph. Aneurysmal dilatation of the azygos vein is uncommon and idiopathic aneurysms are very rare with only a few cases reported in literature. It usually occurs in patients with portal hypertension, heart failure, malformations of the inferior vena cava including partial or total agenesis, or ob-

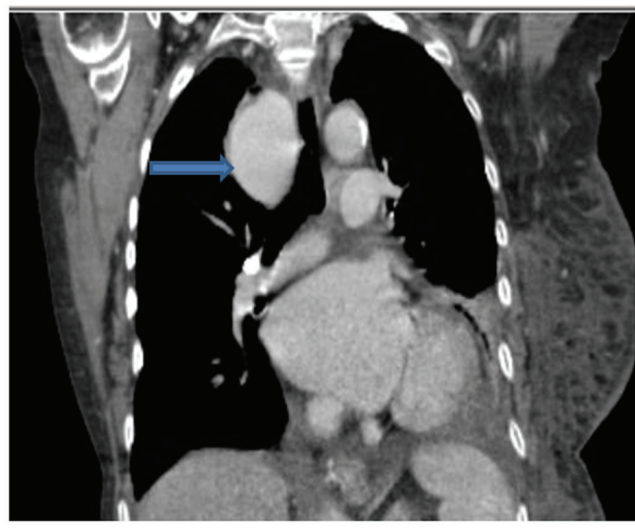

Figure 3. Computed to tomographic scan of chest (coronal section) showing the azygous arch aneurysm.

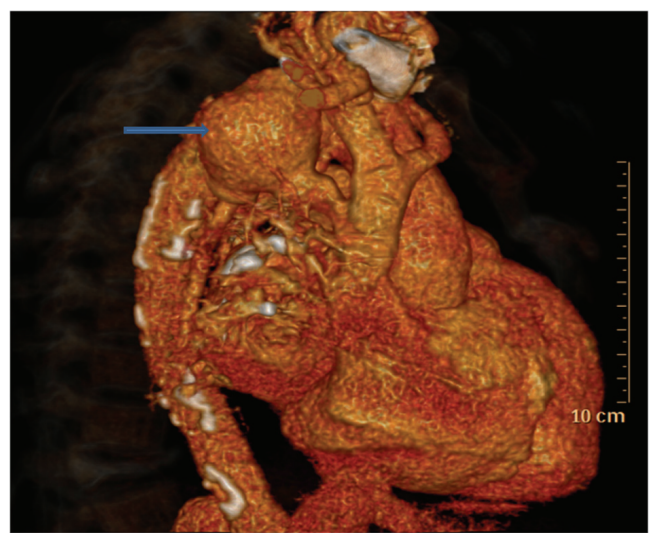

Figure 4. The 3-dimensional CT reconstruction image showing the Azygos aneurysm (arrow).

struction of the inferior vena cava by a tumor or other causes. These dilatations are directly related to high blood flow status of the azygos vein system as a collateral [6]. However, in case of a saccular aneurysm the etiology is congenital and stems from a developmental malformation [4].

During the third and fourth weeks of gestation the cardinal vein system develops. This system consists of paired anterior and posterior cardinal veins which unite to form a short common cardinal vein. The anterior cardinal vein gives rise to the subclavian, the internal jugular, the brachiocephalic vein, and the superior vena cava. The posterior cardinal veins are replaced by two additional pairs of veins, the subcardinal and the supracardinal veins (which gives rise to a portion of the inferior vena cava and the azygos system). A segment of the right supracardinal vein anastomoses with a part of the superior vena cava (derived from the anterior cardinal vein) so that the azygos drains into the right atrium via the superior vena cava. In a case of a saccular aneurysm, a remnant of either the right posterior cardinal vein, subcardinal vein, or primitive subclavian vein, which empties into the transverse part of the azygos vein, may be the origin [11].

In patients with an azygos venous aneurysm a frontal chest radiograph may demonstrate a prominent azygos vein or an abnormal mediastinal density. On a frontal chest radiograph, characteristically, the size of the aneurysm changes with the respiratory movements (especially with Valsalva maneuver). In the past, venography used to be performed to suggest the diagnosis [2-5]. Nowadays, dynamic enhanced CT scanning and MRI not only provide non-invasive methods for the evaluation of vascular abnormalities, but can also rule out other entities which may produce enlargement of the azygos vein $[12,13]$. Watanabe et al. reported that there was great enhancement in the early phase at the site near the azygos arch, but there was little enhancement at the site away from the arch in dynamic enhanced-CT [14]. In our report, a dynamic enhanced-CT showed similar features. Biopsy is absolutely contraindicated because of its extremely high risk 
of life-threatening bleeding.

An azygos vein aneurysm is usually asymptomatic and often detected incidentally on chest radiographs obtained for another purpose. As the aneurysm enlarges, it may lead to pressure effects on adjacent tissues, such as obstruction of the superior vena cava and compression of the right main bronchus or the right upper lobe bronchus [2]. Theoretically, pulmonary thrombosis may be caused by intraluminal thrombus, and rupture of the aneurysm may occur [15]. An appropriate therapeutic strategy is not clear. Except for the report by Jain and Blebea showing posttraumatic pseudoaneurysm of the azygos vein, there have been no reports of the aneurysm leading to rupture and hemorrhage [16]. In the scenario that a preoperative diagnosis of azygos vein aneurysm can be made, no definite cause or symptom can be found, and when the aneurysm is not large and has no intraluminal thrombus, conservative management with follow-up is suggested [17]. In the case presented there has been no change in the size of the aneurysm on follow up CT Scan after one year.

Most azygos vein aneurysms reported previously were resected by thoracotomy. The indications for surgical resection are not yet clear. Surgical treatment may prevent complications arising from the aneurysm, such as pulmonary embolism, venous rupture, and pressure effects on adjacent structures. In total, there have been 5 cases reported with thrombosis in the aneurysm to date, including a case of a large thrombosed azygos vein aneurysm that caused a massive bilateral pulmonary embolism [18]. An enlarged azygos vein aneurysm should be resected because of its compression of adjacent structures, such as the trachea, the right main bronchus, and the SVC. Endovascular treatment has recently been developed and should be considered as an alternative to surgical treatment because of its microinvasiveness and effectiveness [19].

\section{Conclusion}

Although this entity is very rare, we believe that it should be borne in mind for the differential diagnosis of a mediastinal mass. Moreover, it should be stressed that, for an accurate diagnosis, invasive tests are not necessary. Follow up of this lesion is important as an azygos vein aneurysm may grow and surgical intervention may be necessary [20]. In our patient there has been no change in the size of the aneurysm over a one year follow up and she continues to be asymptomatic.

\section{References}

1. Rockoff SD, Druy EM. Tortuous azygos arch simulating a pulmonary lesion. AJR Am J Roentgenol. 1982;138(3):577-579.

2. Seebauer L, Prauer HW, Gmeinwieser J, Sebening F.
A mediastinal tumor simulated by a sacculated aneurysm of the azygos vein. Thorac Cardiovasc Surg. 1989;37(2):112-114.

3. Stern WZ, Bloomberg AE. Idiopathic azygos phlebectasia simulating mediastinal tumor. Radiology. 1961;77:622-625.

4. Kurihara Y, Nakajima Y, Ishikawa T. Case report: saccular aneurysm of the azygos vein simulating a paratracheal tumour. Clin Radiol. 1993;48(6):427-428.

5. Magbitang MH, Hayford FC, Blake JM. Dilated azygos vein simulating a mediastinal tumor. Report of a case. N Engl J Med. 1960;263:598-600.

6. Lena H, Desrues B, Heresbach D, Quinquenel ML, Corbineau H, Rioux C, Delaval PM. Azygos vein aneurysm: contribution of transesophageal echography. Ann Thorac Surg. 1996;61(4):1253-1255.

7. T MethaM, TowersM. Computed tomography appearance of idiopathic aneurrysm of the azygos vein. J Can Assoc Radiol 1996;47:288-290.

8. Podbielski FJ, Sam AD, 2nd, Halldorsson AO, IashaSznajder J, Vigneswaran WT. Giant azygos vein varix. Ann Thorac Surg. 1997;63(4):1167-1169.

9. Walker WA. Aneurysm of the Azygos Vein, Etiology Undetermined. Am J Roentgenol Radium Ther Nucl Med. 1963;90:575-577.

10. Juraszynski Z, Zielinski D, Burakowska B. Trivial chest injury leading to azygos vein pseudoaneurysm. Am J Med. 2010;123(10):e9-e10.

11. Willians PL. Embryology and development. In: Gray's anatomy: the anatomical basis of medicine and surgery. 38th edition. New York: Churchill Livingstone, 1995: 324-327

12. Lee DH, Keum DY, Park CK, Kim JB, Rho BH. Azygos Vein Aneurysm - A Case for Elective Resection by Video-assisted Thoracic Surgery. Korean J Thorac Cardiovasc Surg. 2011;44(4):304-306.

13. Moncada R, Demos TC, Marsan R, Churchill RJ, Reynes C, Love L. CT diagnosis of idiopathic aneurysms of the thoracic systemic veins. J Comput Assist Tomogr. 1985;9(2):305-309.

14. Watanabe A, Kusajima K, Aisaka N, Sugawara H, Tsunematsu K. Idiopathic saccular azygos vein aneurysm. Ann Thorac Surg. 1998;65(5):1459-1461.

15. Icard P, Fares E, Regnard JF, Levasseur P. Thrombosis of an idiopathic saccular azygos aneurysm. Eur J Cardiothorac Surg. 1999;15(6):870-872.

16. Jain A, Blebea JS. Post-traumatic pseudoaneurysm of the azygous vein in a patient with azygous continuation. J Comput Assist Tomogr. 1994;18(4):647-648.

17. He J, Mao H, Li H, Zhu B, Chen J, Zhou Z. A case of idiopathic azygos vein aneurysm and review of the literature. J Thorac Imaging. 2012;27(4):W91-93.

18. D'Souza ES, Williams DM, Deeb GM, Cwikiel W. Resolution of large azygos vein aneurysm following 
stent-graft shunt placement in a patient with EhlersDanlos syndrome type IV. Cardiovasc Intervent Radiol. 2006;29(5):915-919.

19. Gnanamuthu BR, Tharion J. Azygos vein aneurysm--a case for elective resection. Heart Lung Circ.
2008;17(1):62-64.

20. Gallego M, Mirapeix RM, Castaner E, Domingo C, Mata JM, Marin A. Idiopathic azygos vein aneurysm: a rare cause of mediastinal mass. Thorax. 1999;54(7):653655. 